\title{
Hot Immunological Topics in HIV Infection
}

Juan A. Pineda ${ }^{1}$, José Alcamí ${ }^{2}$, José R. Blanco ${ }^{3}$, Julià Blanco4, Vicente Boix ${ }^{5}$, José L. Casado ${ }^{6}$, Juan C. López-Bernaldo de Quirós ${ }^{7}$, Josep M. Llibre ${ }^{8}$

${ }^{1}$ Unit of Infectious Diseases, Hospital Universitario de Valme, Seville, Spain ${ }^{2}$ AIDS Immunopathogenesis Unit, Instituto de Salud Carlos III, Madrid, Spain

${ }^{3}$ Division of Infectious Diseases. Hospital San Pedro-CIBIR. Logroño, Spain

${ }^{4}$ Fundació IrsiCaixa, Institut Germans Trias i Pujol, Badalona, Spain

${ }^{5}$ Unit of Infectious Diseases. Hospital General de Alicante. Alicante, Spain

${ }^{6}$ Department of Infectious Diseases, Hospital Ramón y Cajal, Madrid, Spain

${ }^{7}$ Unit of Infectious Diseases, Hospital General Gregorio Marañón, Madrid, Spain

${ }^{8}$ Fundació Lluita contra la SIDA, Hospital Universitari Germans Trias i Pujol, Badalona, Spain

\begin{abstract}
Incomplete immune reconstitution and persistent immune system hyperactivation in spite of highly active antiretroviral therapy continue to be a challenge. Both facts may lead to an increased risk for AIDS-defining and non AIDS-defining clinical conditions and may also promote atherogenesis and liver fibrogenesis in HIV and hepatitis $\mathrm{C}$ virus-coinfected patients. In this article, the use of new markers to assess immune reconstitution and immune activation and the incidence and clinical consequences of immunediscordant response to antiretroviral therapy are addressed. Likewise, the impact of immune dysfunction on atherogenesis and liver fibrogenesis are reviewed. Finally, it is discussed whether therapy with drugs belonging to the family of CCR5 inhibitors may provide additional immunological benefit in HIV-infected patients.
\end{abstract}

Keywords: HIV; AIDS; Hepatitis C virus; Atherosclerosis; CD4+ cells; CCR5 inhibitors

\section{Introduction}

Fifteen years after highly active antiretroviral therapy (HAART) became the standard of care in HIV infection, a few issues regarding the immunological effects of such therapy have been clarified, but still many points remain incompletely understood. Thus, we have learnt that the use of a combination of potent antiviral drugs leads to a reconstitution of the immune system, which in the short- and in the mid-term is sufficient to radically increase the life expectancy and to markedly reduce the incidence of opportunistic events [1]. However, a non-negligible proportion of patients do not achieve a significant gain of CD4+ cell count, which raises concerns on the risk of AIDS-related and non-AIDS related events in this setting, particularly in the longterm [2]. In addition, HIV-infection causes a chronic hyperactivation of the immune system, which may have an impact on the evolution of disorders with an inflammatory basis, as liver fibrosis or atherogenesis. On the other hand, a number of immunological markers, that may be used to assess to what extent the immune system is reconstituted, have been developed in the last few years, although their potential for being embodied into the routine clinical practice is not well-defined. Finally a new family of antiretroviral drugs, the CCR5 antagonists, has been incorporated into the available armamentarium against HIV. These drugs may inhibit the joining between chemokines and CCR5, having, therefore, immunomodulatory and antiinflammatory effects [3]. Furthermore, therapy with Maraviroc (Selzentry ${ }^{\mathrm{mix}}$, ViiV Helthcare) leads to greater and faster CD4+ cell gain $[4,5]$. These additional benefits on immune reconstitution could open new prospects in the therapy of HIV infection.

The purpose of this article is to review the potential role of new markers in measuring the immune response to HAART, the mechanism and clinical implications of failure to achieve immune reconstitution despite virological control, the impact that immune damage associated to HIV infection may have on the progression of liver fibrosis and atherosclerosis and the role that CCR5 antagonists may play in improving the immunological response to HAART.

\section{Immunological markers with potential clinical usefulness}

Destruction of CD4+ lymphocytes is the key pathogenic fact of HIV infection. CD4+ cell depletion initiates at an early stage of infection and activated CD4+ cells, mainly those located in the mucosa-associated lymphoid tissue, represent the preferential target of HIV in the acute and chronic phase of infection [6]. Both direct cytopathic effect of infected CD4+ lymphocytes [7] and apoptosis-mediated mechanisms are involved in CD4+ destruction $[8,9]$. Because activated cells are preferential targets for HIV infection and replication, HIV specificCD4+ lymphocytes, which become activated by viral antigens, are preferentially killed by the virus [10]. This fact shortens the life span of HIV-specific CD4+ T cells in vivo and decreases the capacity of the immune system to recognize and destroy HIV-infected cells [11]. Furthermore, once HLA-restricted immune responses are triggered, infected $\mathrm{CD} 4+$ lymphocytes become targets that are recognized and killed by HIV-specific CD8+ lymphocytes [12]. Because CD4+ lymphocytes play a central role in the regulation and activation of the immune system [13], their destruction by HIV through different mechanisms has a profound impact in the differentiation and function of other lymphocyte subsets.

In addition to CD4 depletion, recent results show the crucial role of continuous immune activation in the pathogenesis of AIDS [14]. In the chronic phase of infection, HIV establishes a vicious cycle that in turn

${ }^{*}$ Corresponding author: Dr. Juan A. Pineda, Unidad Clínica de Enfermedades Infecciosas. Hospital Universitario de Valme, Avda, Bellavista s/n. 41014-Seville Spain, Tel: +34 955015864; Fax: +34 955015795; E-mail: japineda@telefonica.net

Received January 18, 2011; Accepted February 24, 2011; Published February 25, 2011

Citation: Pineda JA, Alcamí J, Blanco JR, Blanco J, Boix V, et al. (2011) Hot Immunological Topics in HIV Infection. J AIDS Clinic Res 2:118. doi: 10.4172/255-6113.1000118

Copyright: @ 2011 Pineda JA, et al. This is an open-access article distributed unde the terms of the Creative Commons Attribution License, which permits unrestricted use, distribution, and reproduction in any medium, provided the original author and source are credited. 
increases the activation of the immune system by different mechanisms. On the one hand, the destruction of gut-associated lymphoid tissue by HIV increases bacterial translocation, through which bacterial superantigens and wall products activate systemic lymphocytes at multiple levels [15]. On the other hand, antigenemia, due to reactivation of endogenous viruses, can also result in broad activation of T-cell response, all these processes also facilitating the infection of activated CD4+ lymphocytes [16]. Chronic activation of the immune system creates a constant turnover of the system itself that leads to burnout and early senescence of the system. The final result is not only a quantitative change in the CD4+ cell destruction, but malfunction due to poor final differentiation and profound disruption of lymphocyte homeostasis [14]. Increased oxidative stress in non-infected CD4+ cells induced by HIV is an additional mechanism involved in CD4+ cell depletion [17].

Determination of peripheral blood CD4+ levels is the main monitoring tool to assess the prognosis of the patient, to indicate HAART initiation and to evaluate the degree of immune reconstitution following treatment. Serum markers such as $\beta$-2-microglobulin, neopterin or soluble IL2-receptor had little additional predictive power beyond CD4+ cell counts [18]. Besides, classical phenotypic markers of lymphocytic activation such as CD25, DR, CD69, CD38 or soluble cytokines have been proposed as prognostic markers in HIV infection $[19,20]$. However, many of them require in vitro culture and activation of lymphocytes, making these tests unrealistic to be used in the routine follow-up of patients $[21,22]$. In addition, classical markers of lymphocyte activation are relatively nonspecific and may increase due to other infections [23]. Therefore, these parameters are interesting for investigational studies, but of low value in the follow-up of HIVinfected patients in the clinical setting. Specifically, these markers, previously analyzed in studies on HIV progression, have been found to be useful in HIV vaccine trials [24].

Recent findings on the mechanisms of HIV pathogenesis point to the potential utility of several immune markers in the near future. First, CD4+ lymphocytes displaying a central memory phenotype, characterized by the expression of the CCR7 receptor together with CD45RA- CD45RO+ CD62L-, has been reported to be preferentially infected by HIV [24]. Second, as a consequence of the persistent immune activation, a premature immune senescence has been described for both CD8+ and CD4+ subpopulations [25]. These cells have reached a terminal differentiation and express the CD57 molecule on their membrane [26], in contrast with CD28+ cells that maintain capacity to activate and to respond to appropriate antigens. Finally, the balance between two different lymphocyte subpopulations, Th17+ and TReg has been found to be relevant in HIV infection [27]. Th17+ lymphocytes are preferentially located in the GALT system and display a strong antiviral activity, which is associated with lack of progression to AIDS [28]. These cells produce IL17 and IL23 and express the chemokine receptor CCR6 and the surface marker CD161. Because interleukin-17 serves to maintain the integrity of the mucosal barrier, loss of Th17+ cells may lead to an increase in microbial translocation. TReg lymphocytes have the capacity to inhibit the activation and proliferation of other lymphocyte subtypes, including Th17+ cell through the production of TGF- $\beta$. This cell subtype displays a CD4+CD25+ FOXP3+ phenotype [29] and its role in HIV infection remains controversial. Consequentely, phenotyping of different subsets of memory cells (CCR7), markers of immune senescence (CD28, CD57) and Th17 (CD161) lymphocytes could provide a more detailed picture on the degree of health or damage of the immune system. If the relevance of these markers to assess the immune status of HIV-infected patients is confirmed in large series of cases, they could be particularly useful in the decision of treatment initiation in HIV-infected individuals.

\section{HAART-associated immune recovery dynamics}

During the first 2 weeks of HAART, there is a quick drop in the plasma HIV viral load following the inhibition of viral replication in productively infected cells. This drop is associated with an increase in the CD4+ lymphocyte count in three different phases [30]. Initially, there is an increase in CD4+ and CD8+ memory cells, due to redistribution of lymphocytes from the lymph nodes. This phase lasts from 2 to 4 weeks and it is followed by a decrease in the immunological activation, along with a diminution of CD4+ and CD8+ cells expressing CD38+ or HLADR antigens, both markers of immunological activation. Finally, after 4 or 6 months of therapy, there is an increase in the naive CD4+ cells which were absent at the beginning of the treatment. In parallel with that expansion, there is a recovery in the functionality of the immune system, and it has been proven that there is a recuperation of the lymphoproliferative responses [31] and in the repertoire of the T-cell receptor [32], along with a restoration of the responses to different antigens, as the tetanus toxoid [33]. Although this restoration decreases the incidence of opportunistic infections and the mortality associated with AIDS, it is unclear how much immune reconstitution is necessary. Some recent works suggest that until the CD4+ cell counts reach 500 cells/ $\mu \mathrm{L}$ for at least 5-7 years, mortality does not match that of HIV non-infected population [34].

\section{Immunodiscordant response}

A number of subjects starting HAART show a discrepancy between plasma viral load response and the CD4+ T-cell recovery. Thus, CD4+ cell count can rise despite persistently detectable plasma viral load (virological nonresponders), or, conversely, it may not increase despite full plasma viral load suppression (immunological nonresponders). There are controversies about the definition of immunological failure. Some authors consider that a CD $4+$ cell count below 200 cells $/ \mu \mathrm{L}$ after 1 year of effective antiretroviral therapy would identify patients with lack of immune recovery. In addition, other studies have defined immune failure as lack of $50 \%$ of increase over the baseline level or a CD4+ cell gain lower than 50-100 cells/ $\mu \mathrm{L}$ within 6 months after starting HAART, since lack of response can be detected earlier using this time limit. However, recent guidelines also include as immunological non-responders those patients who continue below 350-500 cells $/ \mu \mathrm{L}$ after 3-5 years of therapy, as these patients could have a higher risk of progression and death [35]. Immunodiscordant response to HAART, defined as failure to recover CD4+ T cell count above 350-500 cells/ $\mu \mathrm{L}$, has been observed in up to $30 \%$ of virologically suppressed HIVinfected individuals [2].

Defective immune reconstitution may depend on several factors, including previous therapeutic failure, duration of antiretroviral therapy, low CD4+ T-cell count at the initiation of HAART, advanced stage of disease, low adherence to HAART, and previous treatment interruption. However, the nadir CD4+ cell count, a marker of the damage that HIV replication has induced in these patients, is the most consistent factor associated with the lack of recovery, as shown in several large cohort studies [36].

Abundant work aimed at identifying the mechanisms that underlie the lack of immune recovery has shown an accumulation of $\mathrm{T}$ cells in late differentiation stages [37-39], a condition that can be defined as immune exhaustion. Such accumulation of elder CD4+ T cells is due to a significant reduction in thymic output [37-39] and a high level of activation [38,39], which, in turn, induce a premature senescence (40) and an increased sensitivity to programmed CD4+ cell death [37-39]. In fact, the increased CD4+ T cell death by apoptosis appears to be the 
final mechanism of the lack of $\mathrm{T}$ cell recovery [41]. Several reasons may contribute to this general status of immune exhaustion. Lymphoid tissue damage induced by HIV replication may impair proper T cell development [2] and it has been shown to cause permeability of mucosal barriers, increasing blood concentration of bacterial bioproducts, resulting in continuous $\mathrm{CD} 4+\mathrm{T}$ cell activation $[39,42]$. In addition, the exacerbated activation of CD4+ T cells may favor confined HIV replication in lymphoid tissue, which may impair key steps of CD4+ T cell recovery, i. e. thymic output and CD4+ T cell death.

Immunodiscordant response is associated with an increased risk of opportunistic events and death [2]. Because of this, several therapeutic approaches have been tested in order to overcome this condition. Given the predictive value of nadir $\mathrm{CD} 4+\mathrm{T}$ cell count, the most obvious clinical approach to avoid immunodiscordance is early initiation of HAART (CD4+ $>350$ cells $/ \mu \mathrm{L}$ ). Beyond this, early antiretroviral therapy, the management of immunodiscordant patients has limited options. The choice of the antiretroviral drugs might have some effect in the CD4+ cell count recovery. Thus, multiple clinical trials have shown that protease inhibitors increase the CD4+ to a greater extent than non-nucleoside drugs [43]. However, whether this statistically significant benefit translates into a clinical benefit is unknown. In pivotal clinical trials, Maraviroc has also been found to lead to a greater and earlier increase in CD4+ cell counts compared with control groups, both in treatment-naïve and experienced patients, including those who achieved undetectable viral load and those with virological failure $[4,45]$. Because of this, the use of this drug might provide some benefit in the specific setting of patients with immunodiscordant response. In a pilot study, the intensification with maraviroc led to a modest increase in CD4+ cell counts, a decreased activation of CD4+ and CD8+ cells, as measured by the proportion of CD38+ and HLA-DR+/ CD38+ cells and to a improvement in markers of apoptosis [46]. These analytical benefits should be evaluated in randomized clinical trials. Immune-based approaches, including cytokines (IL-2 or Il-7), have transient positive effects on CD4+ T cell count recovery [47], but no clinically benefit have been demonstrated. Further knowledge of the immunological mechanisms that control CD4+ T cell homeostasis in immunodiscordant patients may help to define new immune-based approaches.

\section{Immunological failure and clinical outcome}

AIDS-related conditions: It is well-known that achieving viral suppression is associated with a marked decrease in the risk of clinical complications, regardless the increase in $\mathrm{CD} 4+$ cell counts. Some studies have even suggested that stopping prophylaxis against Pneumocystis jiroveci in patients below 200 cells $/ \mu \mathrm{L}$ and viral suppression is safe [48]. However, large cohort studies have shown that patients with immunodiscordant response have a 2-8-fold higher risk of opportunistic infections or death, mainly esophageal candidiasis or Pneumocystis jiroveci pneumonia [2,49-51]. In a recent cohort study, among 259 events of opportunistic illness, 214 (83\%) were observed in patients with less than $200 \mathrm{CD} 4+$ cells $/ \mu \mathrm{L}$, and the rate of opportunistic infections in patients on HAART in this population was 24.6 episodes per 1000 patient-years from 1994 to 2007 [51]. Thus, in patients in whom CD4+ T-cell count fails to rise with HAART, therapeutic strategies aimed at increasing these cells and reducing the risk of infections are needed.

Non-AIDS related conditions: The increase in survival that HIV-infected patients have experienced since HAART is available has been related to a lower incidence of AIDS related neoplasms (Kaposi's sarcoma and cerebral lymphoma), and AIDS-defining infections (1).
However, the probability of suffering from non-AIDS related events continues to be high. Among these events, non-AIDS-defining malignancies (NADM) are common [52]. These malignancies include Hodgkin's disease, lung and anal cancer among others.

The risk of NADM could be related to the degree of immunosuppression. In fact, the similar spectrum of NADM among HIV-infected patients and among those who undergo solid organ transplants suggest that immune deficiency, rather than other risk factors, drives this increased risk [53]. However, with the exception of anal cancer and Hodgkin's disease, there is no consistent association between the level of CD4+ cell counts and the incidence of NADM [54]. Coinfection with potentially oncogenic viruses, such as human papillomavirus, Epstein-Barr virus and hepatitis C or B viruses could also increase this risk [55]. Other potential factor for developing NADM is HIV itself, which has oncogenic potential [56]

On the other hand, despite achieving viral suppression with HAART, HIV-infected patients can develop neurocognitive disorders (NCD). NCD may be detected in nearly half of patients and it seems to be also related with immunodeficiency, as they are closely associated with a lower CD4+ nadir [57]. Whereas in the absence of HAART, neurologic impairment is mainly due to neuronal damage, in patients receiving HAART it could be due in part to chronic inflammation secondary to compartmentalized low-level HIV replication or comorbidities $[58,59]$, as well as to the use of a HAART combinations with poor penetration to the central nervous system $[60,61]$.

A better control of NADM and AIDS-related cancers requires an early HIV therapy, started with CD4+ cell counts higher than 350 cells/ $\mu \mathrm{L}$, changes in lifestyle habits (smoke and alcohol cessation), vaccination against oncogenic viruses, such as hepatitis $B$ virus or human papillomavirus, and screening programs for early cancer and NCD detection.

\section{Liver fibrosis and immune system}

Liver fibrosis is a common disorder in all chronic liver diseases, which ends up in cirrhosis and liver failure. The cornerstone of liver fibrosis is the hepatic stellate cell (HSC). In response of hepatocyte necrosis caused by viruses, oxidative stress or bacterial translocation, Kupffer cells are stimulated and proinflammatory cytokines, as well TGF- $\beta$, are released. This leads to HSC activation, with collagen production, a switch to a myofibroblast phenotype, further release of cytokines, chemokines (MCP-1, CCL21, RANTES) and expression of toll-like receptors, which interact with bacterial lipopolysaccharid. In this manner, HSC activation perpetuates and excessive production of extracellular material keeps on [62].

HIV-infected patients may suffer from several disorders causing liver fibrosis, such as chronic hepatitis B or C or non-alcoholic steatohepatitis due to mitochondrial damage caused by nucleoside analogues or insulin resistance. Chronic hepatitis $\mathrm{C}$ is by far the most common cause of liver fibrosis in developed countries. Fibrosis in $\mathrm{HIV} /$ hepatitis $\mathrm{C}$ virus (HCV)-coinfected patients shows an accelerated course [63]. Several studies have demonstrated that a lower CD4+ cell count is associated with faster progression of liver fibrosis and with poorer clinical outcome in HIV/HCV-coinfected patients [64,65]. Accordingly, the greater the CD4+ cell gain after starting antiretroviral therapy, the better the clinical outcome [66]. The reasons why fibrosis has an accelerated progression in HIV/HCV-coinfected patients are not completely understood. Chronic immunoactivaction, increased local production of proinflammatory cytokines and bacterial translocation may play a role. 
CCR5 receptor is important in liver fibrogenesis in $\mathrm{HIV} / \mathrm{HCV}$ coinfected patients. Indeed, CCR5 is strongly expressed by activated HSC. The surface protein gp 120 of HIV induces an increased expression of MCP-1, TIMP-1 and IL-6 in cultured HSC, and these effects are blocked by CCR5 antagonists [67]. Similarly, gp120 and inactivated HIV increase the replication of HCV and the expression of TGF- $\beta$, a strong promoter of fibrogenesis, in vitro. Both effects are inhibited by adding monoclonal antibodies against CCR5 to the cultures [68].

Antiretroviral therapy reduces the activation of the immune system in HIV-infected patients [69], as well as the necroinflammatory activity in the liver in HIV/HCV coinfection [70]. Consequently, the control of HIV replication with antiretroviral therapy slows down fibrosis progression [71] and improves the clinical outcome of liver disease [66]. These effects are likely to be shared by all combinations of HAART. However, inhibitors of the CCR5 could theoretically have a unique effect against fibrosis progression, given the critical role that CCR5 plays in fibrogenesis. Because of this, studies aimed to compare the effects of antiretroviral regimens including CCR5 antagonists on fibrosis progression in HIV/HCV coinfection with that yielded by other drug combinations are warranted.

Immune activation, inflammation and atherosclerosis in HIV-infected patients

Endothelial dysfunction is the link among infection, inflammation and atherosclerosis. Endothelial activation triggers an inflammatory phenomenon which, acting on leucocytes, platelets, coagulation and fibrinolysis, promotes thrombotic occlusion. In HIV-infected patients, high levels of endothelial lesion biomarkers have been consistently detected, including endothelial cell adhesion molecules, as ICAM1, VCAM-1, E-selectin, P-selectin, trombomodulin, class 1 tissue plasminogen activator and class 1 tissue plasminogen activator inhibitor (PAI-1) [72]. HIV infection may induce endothelial dysfunction through several ways. Thus, the virus could infect endothelial cells [73] and endothelial cell activation could also be mediated by cytokines secreted from activated HIV-infected monocytes or directly by gp 120 and Tat HIV proteins (Figure 1).

If HIV infection induces endothelial lesion and promotes atherosclerosis, it makes sense to believe that HIV treatment should diminish the inflammatory component. Thus, HIV-RNA load drop after starting HAART, and the subsequent $\mathrm{T}$ cell function recovery, should eventually reduce HIV-associated endothelial dysfunction. In fact, an improvement in endothelial biomarkers has been reported in patients starting HAART [74] and results of SMART study are in keeping with this finding [75]. However, available data are contradictory and the

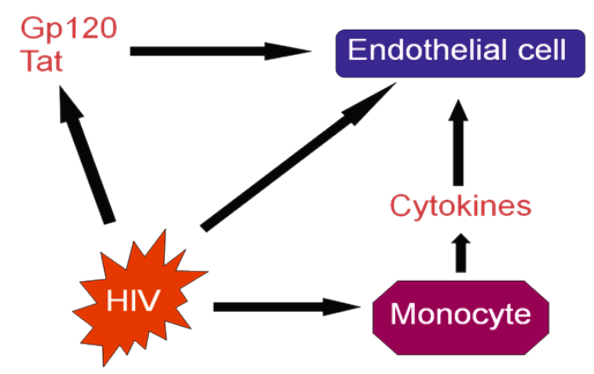

Figure 1: HIV infection may induce endothelial dysfunction through several ways: the virus may infect endothelial cells, cytokines secreted from HIVinfected monocytes could activate endothelial cells or directly by gp120 and Tat HIV proteins. results reported on biomarker changes with HIV treatment have been variable [76]. As a general rule, biomarker levels improve, but they remain higher than in the general population. However, some studies are contradictory and higher levels of P-selectin, t-PA y PAI- 1 have been found in HAART-treated patients, as compared with treatment-naïve patients, with a correlation between endothelial biomarkers and plasma lipids [73]. Similarly, in other studies, an improvement in endothelial biomarkers in patients with antiretroviral treatment has not been observed [77]. In addition, a comparison of patients from the SMART study with general population showed that HIV-infected patients, with or without treatment, had significant higher levels of C-reactive protein, D-dimer and interleukin 6 [78], all of them well-known markers of cardiovascular disease in the general population [79-82].

It is controversial if the effect of different antiretroviral drugs on atherogenesis is distinct. Some observational studies have reported that specific antiretroviral drugs are associated with higher cardiovascular risk [83]. Changes in biomarkers of endothelial function are similar with different antiretroviral combinations, suggesting that endothelial activation is a general consequence of the suppression of HIV replication with HAART, and not a pharmacological effect of specific drugs [84]. However atherosclerosis is a multifactor phenomenon, associated with aspects still incompletely understood. In HIV-infected patients the number of factors influencing atherosclerosis progression is even greater than in patients without HIV infection. In fact, they include not only classical cardiovascular risk factors, but factors directly related to HIV infection and adverse effects of antiretroviral treatment. Because of this, only well-controlled, prospective studies will determine if a specific antiretroviral drug or combination provides added benefit in terms of reducing the progression of atherogenesis.

\section{Immune effects of CCR5 antagonists}

The family of CCR5 antagonists currently comprises Maraviroc, a drug commercially available, and Vicriviroc, whose development has been recently stopped in late phases due to insufficient efficacy [85]. Maraviroc has shown virological superiority over placebo in pretreated patients [4] and non-inferiority compared with Efavirenz in treatmentnaïve individuals [86]. CCR5 antagonists have no activity against CXCR4 tropic strains $[3,44]$. Because of this, the presence of only CCR5 tropic HIV variants must be proven before using these drugs [87-89]. Viral tropism may be determined by phenotypic or genotypic procedures. Genotypic techniques are being increasingly used because they are cheaper, faster and more accessible for local laboratories than phenotypic methods [88]. The response to Maraviroc may be reliably predicted using both phenotypic (Trofile $\mathrm{ES}^{\mathrm{Tu}}$ ) and genotypic procedures, either standard population V3 genotyping or genotyping by massive pyrosequencing with $454[90,91]$.

Maraviroc has been shown to induce a greater and faster CD4+ cell gain than comparators in all studies reported to date. The superior CD4+ cell increase with Maraviroc is independent of achieving plasma HIV-RNA levels less than 50 copies/mL or not in pretreated patients (Figure 2), a finding that has only been proven so far with Maraviroc $[45,86,92]$. The higher CD4+ cell count with Maraviroc is evident at week 4 of therapy [5]. The difference with comparators is greater in pretreated patients $(79$ cells $/ \mu \mathrm{L}$ at week 48$)$ than in treatment-naïve subjects [30 cells/ $\mu \mathrm{L}$ ( $95 \% \mathrm{CI}: 10-51$ cells $/ \mu \mathrm{L})$ at week 48 , and 41 cells/ $\mu \mathrm{L}$ (95\% CI: 17-65) at week 96] [44,45,86]. A metanalysis of all pivotal clinical trials with Maraviroc as salvage therapy has confirmed that this drug causes a significantly higher CD4+ cell gain than comparators [32 cells/ $\mu \mathrm{L}$ (95\% CI: 19-54)] [93]. 


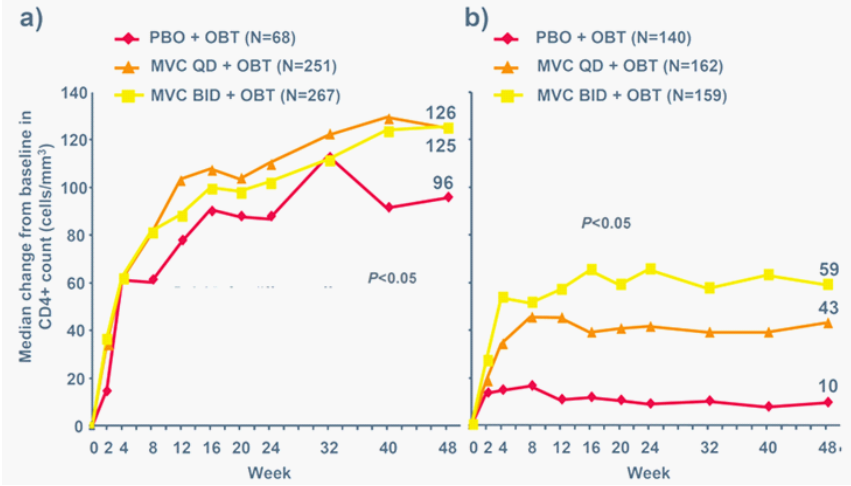

Figure 2: CD4+ T-cell count rises in MOTIVATE 1 and 2 clinical trials in subjects who a) achieved plasma HIV-1-RNA $<50 \mathrm{copies} / \mathrm{mL}$ at least once and, b) never achieved HIV-1-RNA $<50$ copies $/ \mathrm{mL}$. Longitudinal model of CD4+ cell count over time, with week, treatment, and week/treatment interaction as model terms; LOCF used for missing values. MVC: Maraviroc; PBO: placebo; OBT: optimized background treatment. $\mathrm{P}<0.05$ for different effect of treatment over time, combined MVC arms vs PBO (5).
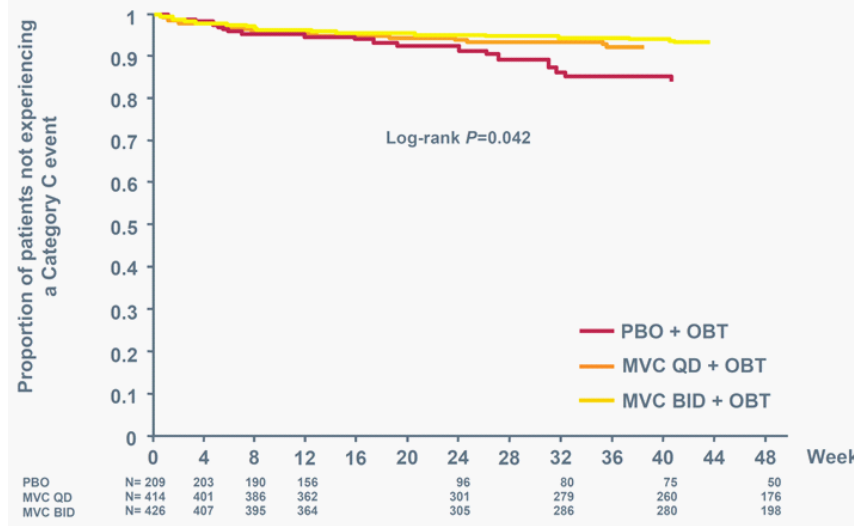

Figure 3: Significantly longer time to occurrence of category $C$ events over time in patients receiving Maraviroc (MVC) vs placebo (PBO), both plus optimized background therapy (OBT), in the MOTIVATE 1 \& 2 clinical trials (combined analysis). Cox proportional hazard regression model using the following variables: baseline HIV-1 RNA, time-dependent CD4+ cell count values, age, gender, and treatment group (MVC arms vs PBO).

The mechanisms by which Maraviroc leads to a greater CD4+ cell recovery are not completely understood. A redistribution of specific CD4+ populations, a blockade of gp120-mediated apoptosis as well as a reduction of immune activation have been proposed as underlying mechanisms for this fact [87]. Indeed, the early decrease in CD4+ and CD8+ activated cells that has been observed in treatment-naïve and pretreated patients who started Maraviroc parallels with CD4+ cell recovery $[45,92]$.

The clinical significance of this greater CD4+ cell increase in patients receiving Maraviroc is not completely clarified. However, some data suggest that it may be relevant. Thus, in severely immunosuppressed patients who start therapy, either treatment-naïve or pretreated, the longer the time with a CD $4+$ cell count below 50 or $100 / \mu \mathrm{L}$, the higher the risk of serious opportunistic events [94]. In the MOTIVATE clinical trials, patients included in the Maraviroc arm showed a CD4+ cell count $50 / \mu \mathrm{L}$ over the comparator arm at week 4 and had a significantly lower rate of opportunistic events (Figure 3) [45]. In this study, each increase of $50 \mathrm{CD} 4+$ cells reduced the risk of a new AIDS-defining event with a hazard ratio of 0.679 . In the same line, in the MERIT clinical trial, which included treatment-naïve patients, the frequency of NADM and AIDS-defining events was lower in patients treated with Maraviroc than in those receiving Efavirenz (1.7\% vs. $3.3 \%$ ), although in this case the differences were not statistically significant, at least in part due to a low number of events in both arms [86].

\section{Conclusions}

In spite of the fact that HAART allows the reconstitution of the immune system, which changes drastically the natural history of HIVinfection, up to $30 \%$ of patients do not achieve a significant gain of $\mathrm{CD} 4+$ cell counts. This failure to reconstitute the immune system is associated with an increased incidence of AIDS-defining conditions. In addition, the incidence of NADM, NCD, atherosclerosis and liver disease, mainly viral hepatitis-related liver diseases, continues to be high in HIV-infected patients under HAART. Chronic activation of immune system, which is not completely reversed by HAART, as well as persistent immune deficiency, may contribute to the emergence as persistence of these disorders. New immune markers that may easily and reliably detect the residual immune damage are required and could be available shortly. Finally, CCR5 antagonists, a new family of antiretroviral drugs, may provide added benefits in terms of CD4+cell gain, reduction of immune activation and inflammatory activity. Studies aimed to assess the effects of these drugs in patients with immune failure, as well as on the incidence on AIDS and non-AIDS events, liver fibrosis and atherosclerosis progression are clearly needed.

\section{Acknowledgments}

We are grateful to Rocio de Jorge-Arellano for her helpful comments, as well as FIPSE foundation and Spanish AIDS Research Network (ISCIII-RETIC RD06/0006) for financial support. Juan A. Pineda is the receptor of an intensification grant from the Fundación Progreso y Salud of the Consejería de Salud de la Junta de Andalucía (Reference Al-0021).

\section{Conflict of Interests}

Juan A. Pineda reports having received consulting fees from GlaxoSmithKline Bristol-Myers Squibb, Abbott Pharmaceuticals, Gilead, Merck Sharp \& Dome Schering-Plough, Janssen Cilag and Boehringer-Ingelheim, research support from GlaxoSmithKline, Roche, Bristol-Myers Squibb, Schering-Plough, Abbott Pharmaceuticals and Boehringer- Ingelheim and lecture fees from GlaxoSmithKline Roche, Abbott Pharmaceuticals, Bristol-Myers Squibb, Gilead, Merck Sharp \& Dome, Janssen Cilag, 372 Boehringer Ingelheim and Schering-Plough.

Josep M Llibre has received research funding, consultancy fees or lecture sponsorships from Abbott Laboratories, Boehringer-Ingelheim, Gilead Sciences, GlaxoSmithKline, Janssen-Cilag, MSD, Pfizer, Roche and ViiV Healthcare.

José Ramón Blanco has received research funding from Gilead and consultancy fees or lecture sponsorships from Bristol-Myers Squibb, Gilead Sciences, GlaxoSmithKline, Janssen-Cilag, MSD, and ViiV Healthcare.

Vicente Boix has received research funding, consultancy fees or lecture sponsorships from Abbott Laboratories, Boehringer-Ingelheim, Gilead Sciences, GlaxoSmithKline, Janssen-Cilag, MSD, Pfizer, Roche and ViiV Healthcare.

\section{All other Authors}

None to declare.

\section{References}

1. Hogg R, Lima V, Estrellas JA, Grabar S, Battegay M, et al. (2008) Life expectancy of individuals on combination antiretroviral therapy in high-income countries: A collaborative analysis of 14 cohort studies. Lancet 372: 293-299.

2. Gazzola L, Tincati C, Bellistrì GM, Monforte A, Marchetti G (2009) The absence of CD4+ T cell count recovery despite receipt of virologically suppressive highly active antiretroviral therapy: Clinical risk, immunological gaps, and therapeutic options. Clin Infect Dis 48: 328-337.

3. MacArthur RD, Novak RM (2008) Reviews of anti-infective agents: Maraviroc: The first of a new class of antiretroviral agents. Clin Infect Dis 47: 236-241. 
Citation: Pineda JA, Alcamí J, Blanco JR, Blanco J, Boix V, et al. (2011) Hot Immunological Topics in HIV Infection. J AIDS Clinic Res 2:118. doi:10.4172/2155-6113.1000118

Page 6 of 8

4. Gulick RM, Lalezari J, Goodrich J, Clumeck N, DeJesus E, et al. (2008) Maraviroc for previously treated patients with R5 HIV-1 infection. N Engl J Med 359:1429-1441.

5. Asmuth D, Goodrich J, Cooper D, Haubrich R, Rajicic N, et al. (2008) CD4+ cell restoration after 48 weeks in the Maraviroc treatment-experienced trials MOTIVATE 1 and 2. J Acquir Immune Defic Syndr 54: 394-397.

6. Mehandru S, Poles MA, Tenner-Racz K, Horowitz A, Hurley A, et al. (2004) Primary HIV-1 infection is associated with preferential depletion of CD4+ T lymphocytes from effector sites in the gastrointestinal tract. J Exp Med 200: 761-770

7. Mehandru S, Poles MA, Tenner-Racz K, Manuelli V, Jean-Pierre P, et al. (2007) Mechanisms of gastrointestinal CD4+ T-cell depletion during acute and early human immunodeficiency virus type 1 infection. J Virol 81: 599-612.

8. Zhou Y, Shen L, Yang HC, Siliciano RF (2008) Preferential cytolysis of peripheral memory CD4+ T cells by in vitro X4-tropic human immunodeficiency virus type 1 infection before the completion of reverse transcription. J Virol 82: 9154-9163.

9. Finkel TH, Tudor-Williams G, Banda NK, Cotton MF, Curiel T, et al. (1995) Apoptosis occurs predominantly in bystander cells and not in productively infected cells of HIV-and SIV -infected lymph nodes. Nat Med 1: 129-134.

10. Douek DC, Brenchley JM, Betts MR, Ambrozak DR, Hill BJ, et al. (2002) HIV preferentially infects HIV-specific CD4+ T cells. Nature 417: 95-98.

11. Brenchley JM, Ruff LE, Casazza JP, Koup RA, Price DA, et al. (2006) Preferential infection shortens the life span of human immunodeficiency virusspecific CD4+ T cells in vivo. J Virol 80: 6801-6809.

12. Goonetilleke N, Liu MKP, Salazar-Gonzalez JF, Ferrari G, Giorgi E, et al. (2009) The first $T$ cell response to transmitted/founder virus contributes to the control of acute viremia in HIV-1 infection. J Exp Med 206: 1253-1272.

13. Wan YY (2010) Multi-tasking of helper T cells. Immunology 130: 166-171.

14. Brenchley JM, Price DA, Schacker TW, Asher TE, Silvestri G, et al. (2006) Microbial translocation is a cause of systemic immune activation in chronic HIV infection. Nat Med 12: 1365-1371.

15. Deayton JR, Sabin CA, Johnson MA, Emery VC, Wilson P, et al. (2004) Importance of cytomegalovirus viraemia in risk of disease progression and death in HIV-infected patients receiving highly active antiretroviral therapy. Lancet 363: 2116-2121

16. Fahey JL, Taylor JM, Manna B, Nishanian P, Aziz N, et al. (1998) Prognostic significance of plasma markers of immune activation, HIV viral load and CD4+T-cell measurements. AIDS 12: 1581-1590.

17. Jaworowsi A, Crowe SM (1999) Does HIV cause depletion of CD4+ T cells in vivo by the induction of apoptosis?. Immunol Cell Biol 77: 90-98.

18. Peakman M, Mahalingam M, Pozniak A, McManus TJ, Phillips AN, et al. (1995) Markers of immune cell activation and disease progression. Cell activation in HIV disease. Adv Exp Med Biol 374: 17-26.

19. Bürgisser P, Hammann C, Kaufmann D, Battegay M, Rutschmann OT (1999) Expression of CD28 and CD38 by CD8+ T lymphocytes in HIV-1 infection correlates with markers of disease severity and changes towards normalization under treatment. Clin Exp Immunol 115: 458-463.

20. Ostrowski SR, Gerstoft J, Pedersen BK, Ullum H (2003) Impaired production of cytokines is an independent predictor of mortality in HIV-1-infected patients. AIDS 17: 521-530.

21. Altfeld M, Addo MM, Kreuzer KA, Rockstroh JK, Dumoulin FL et al. (2000) $T(H) 1$ to $T(H) 2$ shift of cytokines in peripheral blood of HIV-infected patients is detectable by reverse transcriptase polymerase chain reaction but not by enzyme-linked immunosorbent assay under nonstimulated conditions. J Acquir Immune Defic Syndr 23:287-294.

22. Savarino A, Bottarel F, Malavasi F, Dianzani U (2000) Role of CD38 in HIV-1 infection: an epiphenomenon of T-cell activation or an active player in virus/host interactions?. AIDS 14: 1079-1089.

23. Mothe B, Ibarrondo J, Llano A, Brander C (2009) Virological, immune and host genetics markers in the control of HIV infection. Disease Marker 27: 105-120.

24. Gurunathan S, Habib RE, Baglyos L, Meric C, Plotkin S, et al. (2009) Use of predictive markers of HIV disease progression in vaccine trials. Vaccine 27 : 1997-2015.

25. Appay V, Sauce D (2008) Immune activation and inflammation in HIV-1 infection: causes and consequences. J Pathol 214: 231-241.
26. Deeks SG (2011) HIV infection, inflammation, immunosenescence, and aging Annu Rev Med 62: 141-155.

27. Kanwar B, Favre D, McCune JM (2010) Th17 and regulatory T cells: implications for AIDS pathogenesis. Curr Opin HIV AIDS 5: 151-157.

28. Korn T, Bettelli E, Oukka M, Kuchroo VK (2009) IL-17 and Th17 Cells. Annu Rev Immunol 27: 485-517.

29. Sempere JM, Soriano V, Benito JM (2007) T regulatory cells and HIV infection. AIDS Rev 9: 54-60.

30. Prendergast A, Prado JG, Kang YH, Chen F, Riddell LA, et al. (2010) HIV1 infection is characterized by profound depletion of CD161+ Th17 cells and gradual decline in regulatory T cells. AIDS 24: 491-502.

31. Autran B, Carcelaint G, Li TS, Gorochov G, Blanc C, et al. (1999) Restoration of the immune system with anti-retroviral therapy. Immunol Lett 66: 207-211.

32. Li TS, Tubiana R, Katlama C, Calvez V, Ait Mohand H, et al. (1998) Long-lasting recovery in CD4+T-cell function and viral-load reduction after highly active antiretroviral therapy in advanced HIV-1 disease. Lancet 351: 1682-1686.

33. Chen S, Tuttle DL, Oshier JT, Knot HJ, Streit WJ, et al. (2005) Transforming growth factor-beta1 increases CXCR4 expression, stromal-derived factor1alpha-stimulated signalling and human immunodeficiency virus-1 entry in human monocyte-derived macrophages. Immunology 114: 565-574.

34. Burton CT, Goodall RL, Samri A, Autran B, Kelleher AD, et al. (2008) Restoration of anti-tetanus toxoid responses in patients initiating highly active antiretrovira therapy with or without a boost immunization: An INITIO substudy. Clin Exp Immunol 152: 252-257.

35. Lewden C (2010) Time with CD4+cell count above 500 cells/mm3 allows HIVinfected men, but not women, to reach similar mortality rates to those of the general population: a seven-year analysis. $17^{\text {th }}$ Conference on Retrovirus and Opportunistic Infections. San Francisco, California, USA. Abstract 527.

36. Revised DHHS Guidelines issues (2008) AIDS Pat Car STD 22: 1021.

37. Aiuti F, Mezzaroma I (2006) Failure to reconstitute CD4+ T cells despite suppression of HIV replication under HAART. AIDS Rev 8: 88-97.

38. Benveniste O, Flahault A, Rollot F, Elbim C, Estaquier J, et al. (2005) Mechanisms involved in the low-level regeneration of CD4+ cells in HIV1 -infected patients receiving highly active antiretroviral therapy who have prolonged undetectable plasma viral loads. J Infect Dis 191: 1670-1679.

39. Marchetti G, Gori A, Casabianca A, Magnani M, Franzetti F, et al. (2006) Comparative analysis of T-cell turnover and homeostatic parameters in HIVinfected patients with discordant immune-virological responses to HAART AIDS 20: 1727-1736.

40. Massanella M, Negredo E, Pérez-Alvarez N, Puig J, Ruiz-Hernández R, et al (2010) CD4+T-cell hyperactivation and susceptibility to cell death determine poor CD4+T-cell recovery during suppressive HAART. AIDS 24: 959-968.

41. Molina-Pinelo S, Vallejo A, Díaz L, Soriano-Sarabia N, Ferrando-Martínez $S$, et al. (2009) Premature immunosenescence in HIV-infected patients on highly active antiretroviral therapy with low-level CD4+T cell repopulation. $J$ Antimicrob Chemother 64:579-588.

42. Negredo E, Massanella M, Puig J, Pérez-Alvarez N, Gallego-Escuredo JM, et al. (2010) Nadir CD4+T cell count as predictor and high CD4+T cell intrinsic apoptosis as final mechanism of poor $\mathrm{CD} 4+\mathrm{T}$ cell recovery in virologically suppressed HIV-infected patients: clinical implications. Clin Infect Dis 50: 13001308.

43. Marchetti G, Bellistrì GM, Borghi E, Tincati C, Ferramosca S, et al. (2008) Microbial translocation is associated with sustained failure in CD4+ T-cell reconstitution in HIV-infected patients on long-term highly active antiretrovira therapy. AIDS 22: 2035-2038.

44. Riddler SA, Haubrich R, DiRienzo G, Peeples L, Powderly WG, et al. (2008) Class-sparing regimens for initial treatment of HIV infection. N Engl J Med 358 2095-2106.

45. Saag M, Goodrich J, Fatkenheuer G, Clotet B, Clumeck N, et al. (2009) A double-blind, placebo-controlled trial of Maraviroc in treatment-experienced patients infected with non-R5 HIV-1. J Infect Dis 199:1638-1647.

46. Asmuth DM, Goodrich J, Cooper DA, Haubrich R, Rajicic N, et al. (2010) CD4+ T-cell restoration after 48 weeks in the Maraviroc treatment-experienced trials MOTIVATE 1 and 2. J Acquir Immune Defic Syndr 54: 394-397. 
Citation: Pineda JA, Alcamí J, Blanco JR, Blanco J, Boix V, et al. (2011) Hot Immunological Topics in HIV Infection. J AIDS Clinic Res 2:118. doi:10.4172/2155-6113.1000118

47. Wilkin T, Lalama C, Tenorio A, Landay A, Ribaudo H, et al. (2010) Maraviroc intensification for suboptimal CD4+ cell response despite sustained virologic suppression: ACTG 5256. $17^{\text {th }}$ Conference on Retrovirus and Opportunistic Infections. San Francisco, California. Abstract 285.

48. Levy Y, Lacabaratz C, Weiss L, Viard JP, Goujard C, et al. (2009) Enhanced T cell recovery in HIV-1-infected adults through IL-7 treatment. J Clin Invest 119: 997-1007.

49. D'Egidio GE, Kravcik S, Cooper DL, Cameron DW, Ferguson DA, et al. (2007) Pneumocystis jiroveci pneumonia prophylaxis is not required with a CD4+ T cell count $<200 \mathrm{cells} / \mathrm{ml}$ when viral replication is suppressed. AIDS 21: 1711-1715.

50. Taiwo Bo, Li X, Palella F, Jacobson LP, Margolick JB, et al. (2009) Higher risk of AIDS or death in patients with lower CD4+ cell counts after virally suppressive HAART. HIV Med 10: 657-660.

51. Tan R, Westfall AO, Willig JH, Mugavero MJ, Saag MS, et al. (2008) Clinical outcome of antiretroviral naive HIV infected patients with discordant immunological and virologic responses to highly active antiretroviral therapy. $J$ Acquir Immune Defic Syndr 47: 553-558.

52. Buchacz K, Baker RK, Palella FJ, Chmiel JS, Lichtenstein KA, et al. (2010) AIDS defining opportunistic illnesses in US patients: 1994-2007: A cohort study. AIDS 24: 1549-1554.

53. Crum-Cianflone N, Hullsiek KH, Marconi V, Weintrob A, Ganesan A, et al. (2009) Trends in the incidence of cancers among HIV-infected persons and the impact of antiretroviral therapy: a 20 -year cohort study. AIDS 23: 41-50.

54. Grulich AE, van Leeuwen MT, Falster MO, Vajdic CM (2007) Incidence of cancers in people with HIVIAIDS compared with immunosuppressed transplant recipients: a meta-analysis. Lancet 370: 59-67.

55. Bedimo RJ, McGinnis KA, Dunlap M, Rodriguez-Barradas MC, Justice AC (2009) Incidence of non-AIDS-defining malignancies in HIV-infected versus noninfected patients in the HAART era: impact of immunosuppression. J Acquir Immune Defic Syndr 52: 203-208.

56. Bedimo R (2008) Non-AIDS-defining malignancies among HIV-infected patients in the highly active antiretroviral therapy era. Curr HIVIAIDS Rep 5: 140-149.

57. Nyagol J, Leucci E, Onnis A, De Falco G, Tigli C, et al. (2006) The effects of HIV-1 Tat protein on cell cycle during cervical carcinogenesis. Cancer Biol Ther 5: 684-690.

58. Ellis R, Heaton R, Letendre S, Badiee J, Munoz-Moreno J, et al. (2010) Higher CD4+nadir is associated with reduced rates of HIV-associated neurocognitive disorders in the CHARTER Study: Potential implications for early treatment initiation. $17^{\text {th }}$ Conference on Retrovirus and Opportunistic Infections. San Francisco, California, USA. Abstract 429

59. Anthony IC, Ramage SN, Carnie FW, Simmonds P, Bell JE (2005) Influence of HAART on HIV-related CNS disease and neuroinflammation. J Neuropathol Exp Neurol 64: 529-536.

60. Sinclair E, Ronquillo R, Lollo N, Deeks SG, Hunt P, et al. (2008) Antiretroviral treatment effect on immune activation reduces cerebrospinal fluid HIV-1 infection. J Acquir Immune Defic Syndr 47: 544-552.

61. Letendre S, FitzSimos C, Ellis R, Clifford D, Collier A, et al. (2010) Correlates of CSF Viral Loads in 1,221 Volunteers of the CHARTER Cohort. 17 $7^{\text {th }}$ Conference on Retrovirus and Opportunistic Infections. San Francisco, California, USA. Abstract 172

62. Letendre S, McClernon DR, Ellis RJ, Muñoz-Moreno JA, Way L, et al. (2010) Persistent HIV in the central nervous system during treatments is associated with worse antiretroviral therapy penetration and cognitive impairment. $17^{\text {th }}$ Conference on Retrovirus and Opportunistic Infections. San Francisco, California, USA. Abstract 484b.

63. Brenner DA (2009) Molecular pathogenesis of liver fibrosis. Trans Am Clin Climatol Assoc 120: 361-368.

64. Soriano V, Vispo E, Labarga P, Medrano J, Barreiro P (2010) Viral hepatitis and HIV-coinfection. Antivir Res 85: 303-315.

65. Macías J, Castellano V, Merchante N, Palacios RB, Mira JA, et al. (2004) Effect of antiretroviral drugs on liver fibrosis in HIV-infected patients with chronic hepatitis C: harmful impact of nevirapine. AIDS 18: 767-774.

66. Merchante N, Girón-González JA, González-Serrano M, Torre-Cisneros J, García-García JA, et al. (2006) Survival and prognostic factors of HIV-infected patients with HCV-related end-stage liver disease. AIDS 20: 49-57.
67. Pineda JA, García-García JA, Aguilar-Guisado M, Ríos-Villegas MJ, RuizMorales J, et al. (2007) Clinical progression of hepatitis C virus-related chronic liver disease in human immunodeficiency virus-infected patients undergoing highly active antiretroviral therapy. Hepatology 46: 622-630.

68. Bruno R, Galastri S, Sacchi P, Cima S, Caligiuri A, et al. (2010) Gp120 modulates the biology of human hepatic stellate cells: a link between HIV infection and liver fibrogenesis. Gut 59: 513-520.

69. Lin W, Weinberg EM, Tai AW, Peng LF, Brockman MA, et al. (2008) HIV increases HCV replication in a TGF-beta1-dependent manner. Gastroenterology 134 803-811.

70. Jiménez A, Molero L, Jiménez A, Castañón S, Subirá D, et al. (2002) Role of antiretroviral regimes in HIV-1 patients in reducing immune activation. Immunology 106: 80-86.

71. Mehta SH, Thomas DL, Torbenson M, Brinkley S, Mirel L, et al. (2005) The effect of antiretroviral therapy on liver disease among adults with HIV and hepatitis C coinfection. Hepatology 41: 123-131.

72. Macías J, Berenguer J, Japon MA, Girón JA, Rivero A, et al. (2009) Fas fibrosis progression between repeated liver biopsies in patients coinfected with human immunodeficiency virus/hepatitis C virus. Hepatology 50: 1056-1063.

73. Calza L, Pocaterra D, Pavoni M, Colangeli V, Manfredi R, et al. (2009) Plasma levels of VCAM-1, ICAM-1, Eselectin, and P-selectin in 99 HIV-positive patients versus 51 HIV-negative healthy controls. J Acquir Immune Defic Syndr 50: 430432

74. De Gaetano Donati K, Rabagliati R, lacoviello L, Cauda R (2004) HIV infection, HAART, and endothelial adhesion molecules: current perspectives. Lancet 4 213-222.

75. Monsuez JJ, Charniot JC, Escaut L, Teicher E, Wyplosz B, et al. (2009) HIV associated vascular diseases: structural and functional changes, clinical implications. Int J Cardiol 133: 293-306.

76. Emery S, Neuhaus JA, Phillips AN, Babiker A, Cohen CJ (2008) Major clinica outcomes in antiretroviral therapy (ART)-nalve participants and in those not receiving ART at baseline in the SMART study. J Infect Dis 197: 1133-1144.

77. Mastroianni CM, Lichtner M, Mengoni F, D'Agostino C, d'Ettorre G, et al. (2000) Changes in circulating levels of soluble cell adhesion molecules following highly active antiretroviral treatment of HIV-1-infected patients. Clin Immunol 95: 212-217.

78. Ross AC, Rizk N, O'Riordan MA, Dogra V, El-Bejjani D, et al. (2009) Relationship between inflammatory markers, endothelial activation markers, and carotid intima-media thickness in HIV-infected patients receiving antiretroviral therapy. Clin Infect Dis 49: 1119-1127.

79. Neuhaus J, Jacobs Jr DR, Baker JV, Calmy A, Duprez D, et al. (2010) Markers of Inflammation, Coagulation, and Renal Function Are Elevated in Adults with HIV Infection. J Infect Dis 201:1788-1795

80. Harris TB, Ferrucci L, Tracy RP, Corti MC, Cohen HJ, et al. (1999) Associations of elevated interleukin- 6 and C-reactive protein levels with mortality in the elderly. Am J Med 106: 506-512.

81. Danesh J, Kaptoge S, Mann AG, Sarwar N, Wood A, et al. (2008) Long-term interleukin-6 levels and subsequent risk of coronary heart disease: two new prospective studies and a systematic review. PLoS Med 5: e78.

82. Tzoulaki I, Murray GD, Lee AJ, Rumley A, Lowe GD, et al. (2007) Relative value of inflammatory, hemostatic, and rheological factors for incident myocardial infarction and stroke. The Edinburgh Artery Study. Circulation 115: 2119-2127.

83. Danesh J, Whincup P, Walker M, Lennon L, Thomson A, et al. (2001) Fibrin D-dimer and coronary heart disease: prospective study and meta-analysis. Circulation 103: 2323-2327.

84. Friis Moller N, Reiss O, Sabin CA, Weber R, Monforte A, et al. (2007) Class of antiretroviral drugs and the risk of myocardial infarction. $N$ Engl J Med 356: 1723-1735.

85. Wolf K, Tsakiris DA, Weber R, Erb P, Battegay M (2002) Antiretroviral therapy reduces markers of endothelial and coagulation activation in patients infected with human immunodeficiency virus type 1 . J Infect Dis 185: 456-462.

86. Gathe J, Díaz R, Fatkenheuer G, Zeinecker J, Mak C, et al. (2010) Phase 3 trials with vicriviroc in treatment-experienced subjects demonstrate safety but not significantly superior efficacy over potent background regimens alone. $17^{\text {th }}$ Conference on Retrovirus and Opportunistic Infections. San Francisco, California, USA. Abstract 54LB. 
Citation: Pineda JA, Alcamí J, Blanco JR, Blanco J, Boix V, et al. (2011) Hot Immunological Topics in HIV Infection. J AIDS Clinic Res 2:118. doi:10.4172/2155-6113.1000118

87. Cooper DA, Heera J, Goodrich J, Tawadrous M, Saag M, et al. (2010) Maraviroc versus efavirenz, both in combination with zidovudine-lamivudine, for the treatment of antiretroviral-naive subjects with CCR5-tropic HIV-1 infection. J Infect Dis 201: 803-813.

88. Sax PE (2010) Maraviroc for treatment-naive patients with HIV-1 infection: Is the glass half empty or half full? J Infect Dis 201:797-799.

89. Llibre JM, Schapiro JM, Clotet B (2010) Clinical implications of genotypic resistance to the newer antiretroviral drugs in HIV-1-infected patients with virological failure. Clin Infect Dis 50: 872-881.

90. Soriano V, Perno CF, Kaiser R, Calvez V, Gatell JM, et al. (2009) When and how to use maraviroc in HIV-infected patients. AIDS 23: 2377-2385.

91. McGovern, Zhong X, Mo T, Dong W, Knapp D, et al. (2010) Population-based sequencing of the V3-loop is comparable to the enhanced sensitivity trofile Assay (ESTA) in predicting virologic response to Maraviroc of treatment-naïve patients in the MERIT trial. $17^{\text {th }}$ Conference on Retrovirus and Opportunistic Infections. San Francisco, California, USA. Abstract 92.
92. Swenson L, Dong W, Mo T, Thielen A, Jensen M, et al. (2010) Large-Scale application of "deep" sequencing using 454 technology to HIV tropism screening. $17^{\text {th }}$ Conference on Retrovirus and Opportunistic Infections. San Francisco, California, USA. Abstract 646.

93. Heera J, Ive P, Botes M, Dejesus E, Mayer H, et al. (2009) The MERIT Study of Maraviroc in Antiretroviral-naive patients with R5 HIV-1: 96-week Results. Proceedings of the $5^{\text {th }}$ International AIDS Conference. Cape Town, South Africa. Abstract MOPEB040.

94. Wilkin T, Ribaudo H, Gulick RM (2010) The relationship of CCR5 inhibitors to CD4+cell count changes: A meta-analysis of recent clinical trials. 15th Retroconference on Retroviruses and Opportunistic Infections. Boston, Masachusets, USA. Abstract 800.

95. Zolopa A, Andersen J, Powderly W, Sánchez A, Sanne I, et al. (2009) Early antiretroviral therapy reduces AIDS progression/death in individuals with acute opportunistic infections: a multicenter randomized strategy trial. PLoS ONE 4 e5575. 